\title{
Chapter 1 \\ Near-Field Characterization of Conductive Micro-Resonators for Terahertz Sensing
}

\author{
Irina Khromova ${ }^{12}$, Oleg Mitrofanov ${ }^{13}$ \\ Department of EEE, University College London, UK, i.khromova@ucl.ac.uk \\ Department of Physics, King's College London, UK \\ CINT, Sandia National Laboratories, USA
}

\begin{abstract}
Near-field (NF) terahertz (THz) time-domain spectroscopy (TDS) is an excellent tool for direct studies of THz electromagnetic resonances occurring on a micrometre scale. Micro-resonators are at the heart of numerous promising $\mathrm{THz}$ sensing and detecting solutions, including the metamaterial approach available through functional materials constructed from pre-designed resonant micrometresized 'meta-atoms'. Experimental studies of individual micrometre-scale $\mathrm{THz}$ resonances are essential, yet extremely challenging for the common far-field spectroscopic methods due to extreme sensitivity requirements. NF THz spectroscopy and microscopy are non-contact techniques for spectroscopic studies of individual micro-resonators and mapping the field patterns of $\mathrm{THz}$ resonant modes excited in individual conductive or insulating micro-objects. They give access to essential parameters of micro-resonators, including their resonance frequency, local field enhancement and quality factors. It allows for material and structural characterisation of micro-objects. Using the example of carbon micro-fibres, we show the advantages of NF THz TDS for non-contact THz conductivity probing and direct observation of the fundamental and the third-order surface-plasmon resonance modes in conductive $\mathrm{THz}$ micro-resonators.
\end{abstract}

Keywords: terahertz spectroscopy, metamaterials, carbon fibre, near field

\subsection{Introduction}

To advance terahertz (THz) technology for security applications, including the detection of explosives and drugs through their distinctive $\mathrm{THz}$ and mid-infrared 'signatures' [1], it is essential to develop efficient THz sensors. Extreme sensitivity can be achieved in resonant systems. Micrometre-sized objects, made of con- 
ductive materials, exhibit sharp resonant response at $\mathrm{THz}$ frequencies. They can be used as individual sensors or macro-scale sensing devices, based on metamaterials - artificial structures made of pre-designed resonant 'meta-atoms'.

It is essential to experimentally study and characterise individual microresonators. However, common far-field $\mathrm{THz}$ spectroscopic techniques cannot access the micrometre-scale resonances due to low sensitivity. We demonstrate the successful use of the near-field $\mathrm{THz}$ time-domain spectroscopy (NF THz TDS) for direct experimental characterisation of individual dielectric and conductive microresonators [Khr1].

Through NF THz TDS, we show that at $\mathrm{THz}$ frequencies, carbon micro-fibres (CMFs) [Pon1] exhibit pronounced surface-plasmon resonances. The NF $\mathrm{THz}$ TDS allows for non-contact conductive material characterisation in micrometresized objects, crucial for the practical development of resonance-based devices for the emerging $\mathrm{THz}$ technology for security applications.

\subsection{NF THz TDS for individual micro-objects}

In our experiments, individual micro-resonators (CMFs) are attached to a thin low-density polyethylene (LDPE) film pulled over a sample-holder frame. They are excited by a linearly-polarised broadband $\mathrm{THz}$ pulse $(0.5-2.5 \mathrm{THz})$ generated in a ZnTe nonlinear crystal through optical excitation. The experiments were performed at $22 \pm 1{ }^{\circ} \mathrm{C}$. The enhanced near-field induced in the micro-resonators is detected by photoconductive antenna covered by a metallic screen with a subwavelength aperture [Knab1, Mac1]. We use two different probes with $5 \mu \mathrm{m}$ and $10 \mu \mathrm{m}$ square aperture. Moving the time-delay stage (Fig 1), we perform the near-field spectroscopy in the time-domain.
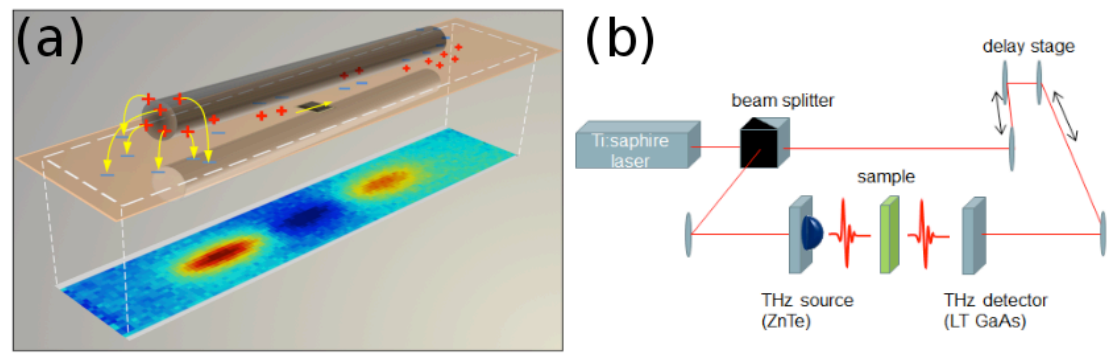

Fig 1 (a) Scheme of the NF THz TDS setup; (b) Schematic image of a CMF probed by the aperture in the metallic plane of the probe. The arrows represent the electric field lines of the complex sample-probe mode. The coloured map is the detected enhanced field distribution measured at a fixed time delay by scanning the sample with respect to the aperture. 


\subsection{Surface-plasmon resonances in THz micro-resonators}

Carbon micro-fibres (CMFs) are milled and chemically-treated carbon fibres of essentially graphitic structure [Pon1] (see Fig 2) produced and supplied by STC of Applied Nanotechnologies. They are $7 \mu \mathrm{m}$ in diameter and their lengths vary between 30-300 $\mu \mathrm{m}$. Depending on the fabrication procedure, the DC resistivity of similar unmilled carbon fibers is of the order of $10 \mu \Omega \times \mathrm{m}$ [Chun1] and is strongly depends on temperature [Spain1]. The plasma frequency of graphite in the conductive plane lies significantly above the $\mathrm{THz}$ region [Tart1, John1] and corresponds to carrier density on the order of $10^{18} \mathrm{~cm}^{3}$.

Given their high conductivity and dimensions comparable to half-wavelength of the working $\mathrm{THz}$ range, CMFs can be considered as half-wave dipole resonators at $\mathrm{THz}$ frequencies [Schel1, Barn1].
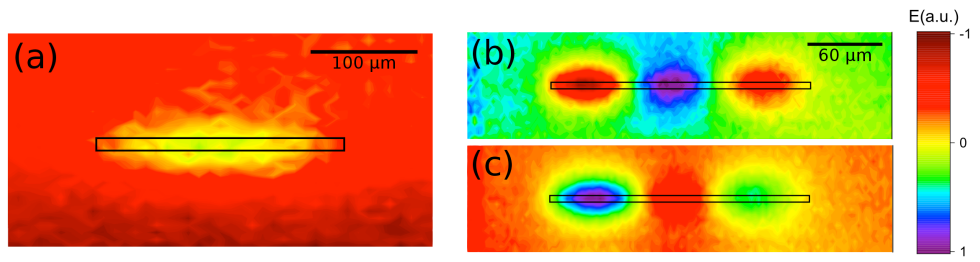

Fig $2(a)$ fundamental and $(b, c)$ third-order surface-plasmon resonance detected in the near-field of a $133 \mu \mathrm{m}$-long and $207 \mu \mathrm{m}$-long CMF, respectively. The maps were acquired at (a) $1.65 \mathrm{ps}$, (b) $1 \mathrm{ps}$, and (c) $1.27 \mathrm{ps}$ after the arrival of the incident $\mathrm{THz}$ pulse.

We performed NF THz TDS on CMFs of different lengths, and were able to detect surface-plasmon resonances (dimensional resonances of induced current) of different modal orders ([Khr1, Mit1]). In the employed setup, the detected signal is proportional to the gradient $d E_{z} / d x$ of the field induced between the conductive $\mathrm{CMF}$ and the detector metallic plane of the THz detector as shown in Fig $1(a)$ [Khr1, Muek1]. As a consequence, when mapping the field of the fundamental resonance mode of a $\mathrm{CMF}$, the maximum signal is measured at the centre of the CMF rather than at its edges.

Figure 2 shows the maps of the detected $\mathrm{THz}$ field obtained through raster scanning the sample with respect to the probe aperture. Figure $2(a)$ shows the map of the detected signal at its highest peak (fixed time delay at $1.65 \mathrm{ps}$ after the arrival of the main pulse) for a $133 \mu \mathrm{m}$-long CMF. This image corresponds to the fundamental surface-plasmon resonance mode of the CMF - half-a-cycle oscillation of the induced current propagating along the structure. Figure $2(b, c)$ shows the maps of the signal detected behind a $207 \mu \mathrm{m}$-long CMF at 1 ps and 1.27 ps after the arrival of the $\mathrm{THz}$ pulse. These maps reflect the detection of a third-order surface-plasmon resonance mode - three halves of the induced current oscillation along the CMFs' length. 


\subsection{Non-contact conductivity probing via NF THz TDS}

While it is possible to get a rough estimate of the average conductivity of a CMF composite via far-field spectroscopic methods, directly measuring the conductivity of a single CMF is a non-trivial task. Through NF THz TDS, we were able to probe the material properties of individual CMFs in a non-contact way.

To access this information about the CMFs' constituent material, we studied the peak amplitude of the enhanced near-field as well resonance frequencies of the fundamental surface-plasmon resonance mode of the CMFs. Both of the two parameters are highly influenced by both the material properties of the resonator and the electromagnetic properties of the environment, including the substrate and the metallic probe effects.

We used full-wave numerical simulations in CST Microwave Studio to predict and separate these effects. The numerical model included the realistic physical parameters of the experimental $\mathrm{THz}$ set-up, including the near-field aperture-based $\mathrm{THz}$ probe.
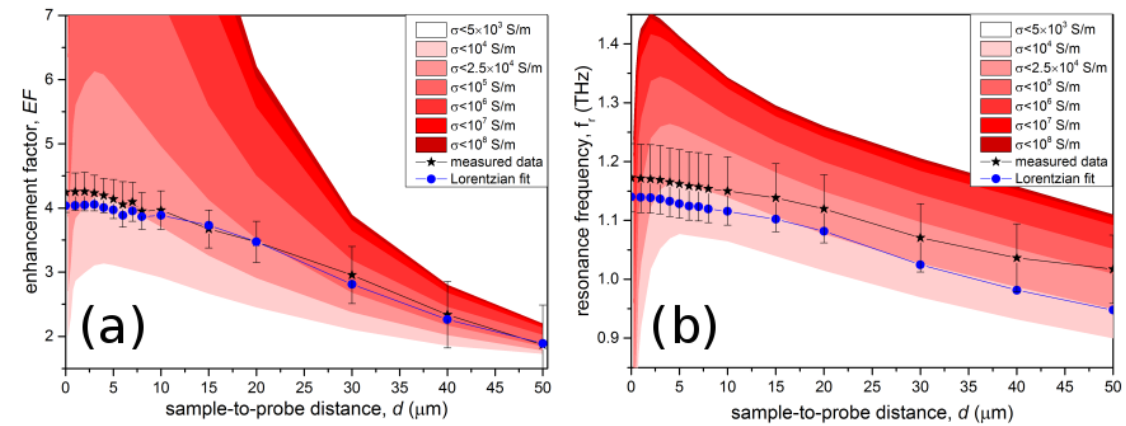

Fig 3 (a) enhancement factor EF and $(b)$ resonance frequency $f_{r}$ of an $80 \mu \mathrm{m}$-long CMF plotted against the sample-to-probe distance. Coloured zones mark numerically calculated areas corresponding to different conductivity bands within the range from $10^{3} \mathrm{~S} / \mathrm{m}$ (material with skin depth comparable to the diameter of the CMFs) to $10^{8} \mathrm{~S} / \mathrm{m}$ (metals). Black curves and symbols represent the measured $E F$ and $f_{r}$, while the blue curves and symbols correspond to $E F$ and $f_{r}$ extracted from Lorentzian fitted experimental spectra. The error bars reflect the effects of noise and the spectral resolution of $58 \mathrm{GHz}$. Reproduced from [Khrl], with the permission of AIP Publishing.

Figure 3 shows the coloured maps of the enhancement factor (amplitude peak of the detected field normalised to the incident field probed by the $\mathrm{THz}$ antenna) and the resonance frequency of the fundamental surface-plasmon resonance. Each colour band represents numerical data obtained for a certain band of CMF conductivity values. Plotting the experimental data against such maps allowed us to estimate the conductivity value corresponding to each of the individual CMFs. We found that the conductivity of the CMFs is $10^{4}-10^{5} \mathrm{~S} / \mathrm{m}$. As the conductivity increases, the areas become narrower. Thus, the proposed method works best for 'weakly' conductive materials, such as doped semiconductors and semimetals. 


\subsection{Substrate-induced resonance frequency shift in CMFs}

The resonance frequency of the surface-plasmon resonances in CMFs are extremely sensitive to the dielectric properties of the surrounding material: we observed how the $12.5 \mu \mathrm{m}$-thick polyethylene substrate with dielectric permittivity $\varepsilon_{s u b}=$ 2.5 shifted the resonance frequency of the CMF fundamental resonance mode by $30 \%$ as compared to the numerically predicted value for an equal, but freestanding CMF (Fig. 4).
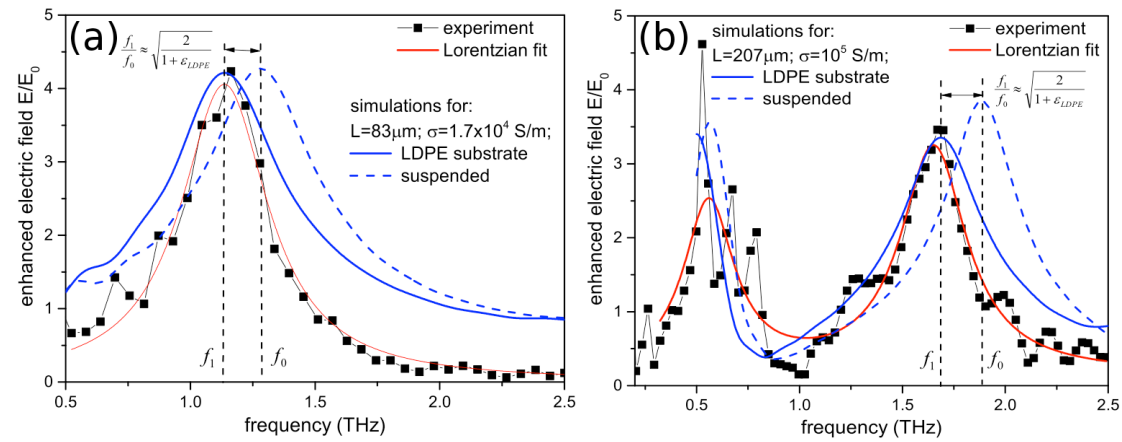

Fig 4 Experimentally obtained enhanced field spectra (black curve and squares) for CMFs placed at $2.5 \mu \mathrm{m}$ from the probe: $(a)$ length $\mathrm{L}=83 \mu \mathrm{m}$; full-wave numerical simulations performed for $\sigma=1.7 \times 10^{4} \mathrm{~S} / \mathrm{m}$; $(b) \mathrm{L}=207 \mu \mathrm{m}$, full-wave numerical simulations performed for $\sigma=10^{5} \mathrm{~S} / \mathrm{m}$. The red curves are the Lorentzian fits. The full-wave numerical simulations were performed for CMFs on LDPE substrates (solid blue curve) and suspended CMF (dashed blue curve). Spectral shift of the resonance frequency is observed due to the change in the permittivity of the sample's environment.

The substrate-induced frequency shift is due to electric field localisation in the immediate vicinity of the CMFs and can be estimated as a factor of $\sqrt{2 /\left(1+\varepsilon_{L D P E}\right)}$. Such behaviour can be utilised for sensing applications, in particular, for probing the dielectric permittivity of substances on a micrometre scale.

\section{Conclusion}

Studying $\mathrm{THz}$ resonant micro-objects in the near-field provides a deep insight into the electromagnetic properties of individual resonators functioning on versatile physical principles. NF THz TDS is an excellent experimental platform for detecting and observing the excitation and evolution of surface-plasmon resonance modes in conductive micro-resonators. We applied this technique to CMFs of different length and observed the excitation of the fundamental as well as the thirdorder resonance mode. 
We demonstrate that NF THz TDS is a promising non-contact method for probing the electric conductivity of individual conductive resonators. The method works best for 'weakly' conductive materials as compared to metals: we successfully measured the conductivity of $10^{4}-10^{5} \mathrm{~S} / \mathrm{m}$ in individual CMFs. It is extremely difficult to perform analogous experiments in the far field using conventional THz TDS systems: assuming their typical beam spot size is approximately 1 $\mathrm{mm}$ in diameter, the presence of a $100 \mu \mathrm{m}$-long CMF would only manifest itself in a mere $\approx 1.5 \%$ intensity dip in transmitted field at the resonance frequency.

We also show that the near-field response of conductive micro-resonators is highly dependent on the environment. We found that the resonance peak of the fundamental and the third-order surface-plasmon resonance mode in conductive CMFs is shifted by the polyethylene substrate by approximately $30 \%$, confirming the sensitivity of CMFs towards the dielectric properties of surrounding materials. This finding can be useful for THz sensing applications.

\section{References}

1. M.F. Pereira, TERA-MIR radiation: materials, generation, detection and applications Opt. Quant. Electron. 46, 491-493 (2014) DOI 10.1007/s11082-0149883-5

2. [Khr1] I. Khromova et al, Dipolar resonances in conductive carbon microfibers probed by near-field terahertz spectroscopy, Appl. Phys. Lett. 107 (2), 021102 (2015) DOI: 10.1063/1.4926628

3. [Pon1] Rus. Fed. Patent N 2570794

4. [Mac1] A. J. Macfaden, J. L. Reno, I. Brener, and O. Mitrofanov, $3 \mu \mathrm{m}$ aperture probes for near-field terahertz transmission microscopy. Appl. Phys. Lett. 104 (1), 011110 (2014) DOI: 10.1063/1.4861621

5. [Mit1] submitted to IEEE

6. [Mit2] O. Mitrofanov, F. Dominec, P. Kužel, J. L. Reno, I. Brener, U-Ch. Chung, C. Elissalde, M. Maglione, and P. Mounaix, Near-field probing of Mie resonances in single $\mathrm{TiO} 2$ microspheres at terahertz frequencies. Opt. Express 22 (19) 23034-23042 (2014) DOI: 10.1364/OE.22.023034

7. [Chun1] D. D. Chung, Carbon Fiber Composites (Butterworth-Heinemann, Boston, 1994)

8. [Spain1] I. L. Spain, K. J. Volin, H. A. Goldberg, and I. L. Kalnin, Unusual electrical resistivity behavior of carbon fibers, Solid State Commun. 45, 817 (1983) DOI: 10.1016/0038-1098(83)90807-4

9. [Knab1] R. Knab, A. J. L. Adam, E. Shaner, H. J. A. J. Starmans, and P. C. M. Planken, Terahertz near-field spectroscopy of filled subwavelength sized apertures in thin metal films, Opt. Express 21, 1101 (2013) DOI: 10.1364/OE.21.001101 
10. [Schel1] S. Schelkunoff and H. Friis, Antennas: Theory and Practice, Applied mathematics series (Wiley, 1952)

11. [Barn1] E. S. Barnard, J. S. White, A. Chandran, and M. L. Brongersma, Spectral properties of plasmonic resonator antennas, Opt. Express, 16, 16529 (2008) DOI: 10.1364/OE.16.016529

12. [John1] L. G. Johnson and G. Dresselhaus, Optical Properies of Graphite, Phys. Rev. B, 7, 2275 (1973) DOI: 10.1103/PhysRevB.7.2275

13. [Tart1] R. C. Tatar and S. Rabii, Electronic properties of graphite: A unified theoretical study, Phys. Rev. B, 25, 4126 (1982) DOI: 10.1103/PhysRevB.25.4126

14. [Muek1] R. Mueckstein, C. Graham, C. C. Renaud, A. J. Seeds, J. A. Harrington, and O. Mitrofanov, Imaging and Analysis of THz Surface Plasmon Polariton Waves with the Integrated Sub-wavelength Aperture Probe, J. Infrared Millimeter Terahertz Waves 32, 1031 (2011) DOI: 10.1007/s10762-011-9811-8 